\title{
Retinal Vascular Changes and Prospective Risk of Disabling Dementia: the Circulatory Risk in Communities Study (CIRCS)
}

\author{
Hiroshige Jinnouchi ${ }^{1}$, Akihiko Kitamura ${ }^{1,2,3}$, Kazumasa Yamagishi ${ }^{2,4}$, Masahiko Kiyama ${ }^{2}$, Hironori Imano ${ }^{1}$, \\ Takeo Okada ${ }^{2}$, Renzhe Cui ${ }^{1}$, Mitsumasa Umesawa ${ }^{4,5}$, Isao Muraki ${ }^{2}$, Mina Hayama-Terada ${ }^{2}$, \\ Ryo Kawasaki ${ }^{6}$, Tomoko Sankai ${ }^{7}$, Tetsuya Ohira ${ }^{8}$ and Hiroyasu Iso ${ }^{1}$; for the CIRCS Investigators \\ ${ }^{1}$ Public Health, Department of Social Medicine, Osaka University Graduate School of Medicine, Osaka, Japan \\ ${ }^{2}$ Osaka Center for Cancer and Cardiovascular Disease Prevention, Osaka, Japan \\ ${ }^{3}$ Research Team for Social Participation and Community Health, Tokyo Metropolitan Institute of Gerontology, Tokyo, Japan \\ ${ }^{4}$ Department of Public Health Medicine, Faculty of Medicine, University of Tsukuba, Ibaraki, Japan \\ ${ }^{5}$ Department of Public Health, Dokkyo Medical University School of Medicine, Tochigi, Japan \\ ${ }^{6}$ Department of Public Health, Yamagata University Graduate School of Medical Science, Yamagata, Japan \\ ${ }^{7}$ Department of Community Health, Faculty of Medicine, University of Tsukuba, Ibaraki, Japan \\ ${ }^{8}$ Department of Epidemiology, Fukushima Medical University School of Medicine, Fukushima, Japan
}

Aim: To investigate the association of retinal vascular changes with a risk of dementia in longitudinal population-based study.

Methods: We performed a nested case-control study of 3,718 persons, aged 40-89 years, enrolled between 1983 and 2004. Retinal vascular changes were observed in 351 cases with disabling dementia (average period before the onset, 11.2 years) and in 702 controls matched for sex, age, and baseline year. Incidence of disabling dementia was defined as individuals who received cares for disabilities including dementia-related symptoms and/or behavioral disturbance. Conditional logistic regression analysis was used to calculate odds ratio (OR) and multivariable adjusted OR (Models 1 and 2) for incidence of disabling dementia according to each retinal vascular change. Regarding confounding variables, Model 1 included overweight status, hypertension, hyperglycemia, dyslipidemia, and smoking status, whereas Model 2 also included incidence of stroke prior to disabling dementia for further analysis.

Results: The proportion of cases (controls) with retinal vascular changes was 23.1 (15.7)\% for generalized arteriolar narrowing, 7.7 (7.5)\% for focal arteriolar narrowing, $15.7(11.8) \%$ for arteriovenous nicking, $10.5(9.3) \%$ for increased arteriolar wall reflex, and $11.4(9.8) \%$ for any other retinopathy. Generalized arteriolar narrowing was associated with an increased risk of disabling dementia: crude OR, 1.66 (95\% confidence interval, 1.19-2.31); Model 1: OR, 1.58 (1.12-2.23); Model 2: OR, 1.48 (1.042.10). The number of retinal abnormalities was associated in a dose-response manner with the risk. Conclusion: Generalized arteriolar narrowing and total number of retinal abnormalities may be useful markers for identifying persons at higher risks of disabling dementia.

See editorial vol. 24: 675-676

Key words: Retinal vascular changes, Retinopathy, Dementia, Cohort studies, Nested case-control studies

Copyright@2017 Japan Atherosclerosis Society

This article is distributed under the terms of the latest version of CC BY-NC-SA defined by the Creative Commons Attribution License.

Address for correspondence: Hiroyasu Iso, Public Health, Department of Social Medicine, Osaka University Graduate School of Medicine, 2-2 Yamadaoka, Suita, Osaka, 565-0871 Japan

E-mail: iso@pbhel.med.osaka-u.ac.jp

Received: July 20, 2016

Accepted for publication: October 16, 2016

\section{Introduction}

Dementia has become a major burden in disabled elderly individuals ${ }^{1,2}$. Microvascular brain damage, such as microinfarction, microhemorrhage, and macrohemorrhage ${ }^{3,4)}$, as well as peripheral arterial stiffness $^{5,6)}$, heighten the risk of cognitive impairment. 


\section{Total number of enrolled persons in the CIRCS $N=3,718$ (1983-2004; 40-89 years at baseline)}

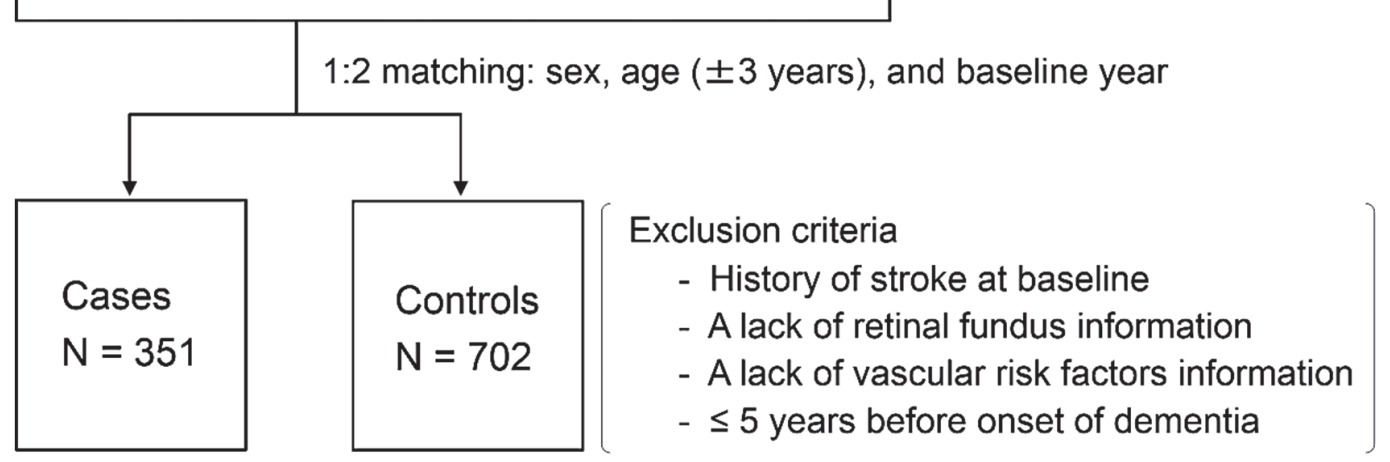

Fig. 1. Diagram of the present study.

Flow diagram of participant enrollment and selection for nested case-control study.

Previous studies have suggested that such brain abnormalities may be associated with retinal vascular changes due to shared embryological, anatomical, and physiological features $^{7-9)}$. In addition, retina is the only location in which it is possible to macroscopically diagnose microvascular abnormalities. Retinal vascular changes, therefore, may serve as risk markers for dementia, but the epidemiological evidence has been limited ${ }^{10)}$.

Several longitudinal population-based studies have investigated the associations between retinal vascular changes and incidence of dementia ${ }^{11}$, or indicators of dementia such as brain imaging abnormalities $^{12-15)}$, cognitive decline ${ }^{16)}$, and disability ${ }^{17)}$, but these results have been inconsistent. In addition, most of these studies investigated the association of retinal vascular changes with the risk of dementia and/or cognitive decline without taking into account incidence of stroke before the onset of dementia. Nevertheless, retinal vascular changes have been shown to be risk markers for incidence of stroke ${ }^{18-20)}$. Therefore, further longitudinal population-based studies are becoming increasingly important in order to confirm these associations while also considering mediation by incidence of stroke.

Our hypothesis was that retinal vascular changes would be associated with an increased risk of disabling dementia and that this association would persist after further adjustment for incidence of stroke before the onset of disabling dementia.

\section{Methods}

\section{Case and Control Identification}

We performed a nested case-control study of 3,718 people, ranging in age from 40 to 89 years, who lived in the Ikawa community in Japan and were enrolled in the Circulatory Risk in Communities Study (CIRCS) between 1983 and 2004 (Fig. 1). Details of the CIRCS protocol have been described elsewhere ${ }^{21-23)}$. Briefly, this cohort was followed via annual cardiovascular risk surveys and surveillance for incidence and mortality of stroke. Surveillance for disabling dementia was also conducted from October 1999 until March 2014.

The criteria of disabling dementia were the same as those of our previous studies ${ }^{21,24-26)}$. Incidence of disabling dementia was defined as individuals who received cares for disability including dementia-related symptoms and/or behavioral disturbance and who were ranked as II or greater on the standardized physicians' classification in Long-Term Care Insurance in Japan. These criteria were validated by comparing with neuropsychiatrists' diagnoses as defined by the International Psychogeriatric Association ${ }^{27}$, which examine five domains of cognitive function: attention, memory, visuospatial function, language, and reasoning. A total of 622 people, aged 65 years or older, were evaluated by neuropsychiatrists and 123 persons were diagnosed with disabling dementia. The calculated sensitivity and specificity values for disabling dementia were $82.9 \%$ and $95.8 \%$, respectively ${ }^{26)}$.

We identified 351 cases with disabling dementia and matched two controls with each case based on sex, age ( \pm 3 years), and baseline year. We excluded 


\section{(A) Generalized arteriolar narrowing}

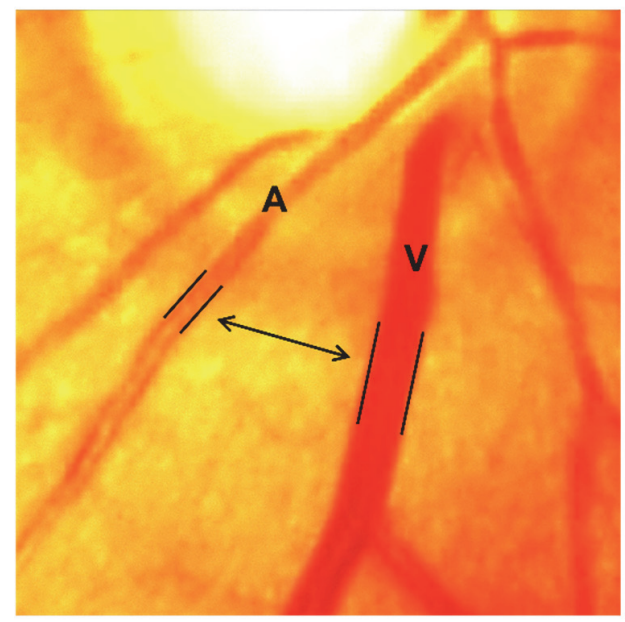

(B) Focal arteriolar narrowing

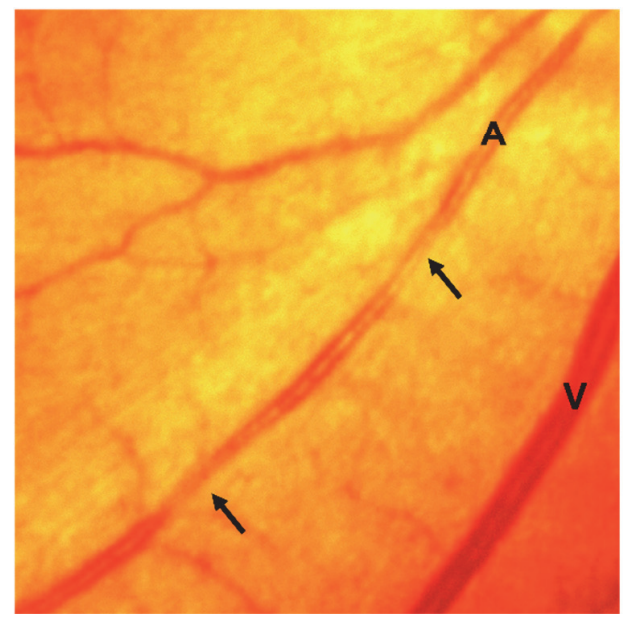

(C) Arteriovenous nicking

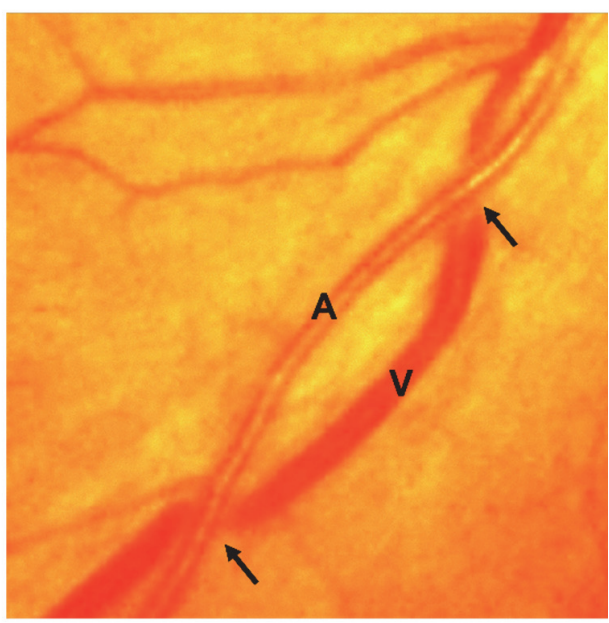

(D) Increased arteriolar wall reflex

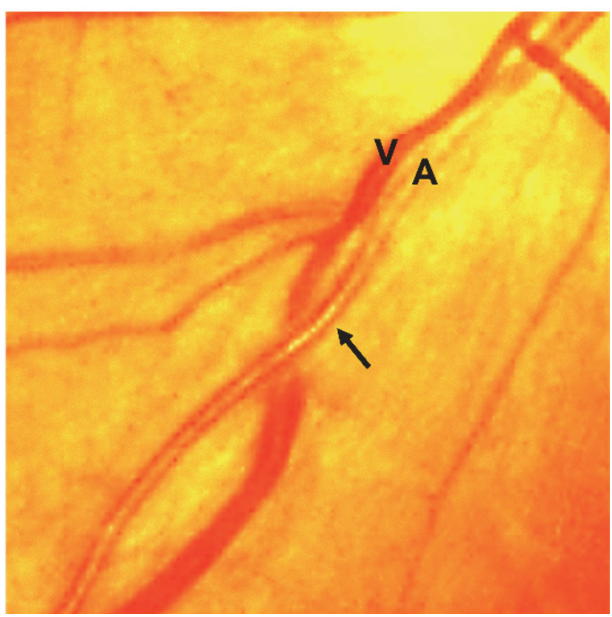

Fig. 2. Example photographs of retinal vascular changes.

A, retinal arteriole; V, retinal venule; Example photographs of retinal vascular changes: (A) Generalized arteriolar narrowing: arteriolar-to-venular diameter ratio of 2:3 or lower; (B) Focal arteriolar narrowing: localized constrictions along the course of arterioles; (C) Arteriovenous nicking: narrowing of a venule as an arteriole crosses over it; and (D) Increased arteriolar wall reflex: an increased light reflex from the central portion of the retinal arteriolar surface; All photographs were modified from Iida and Kitamura (2009) with the authors' permission $^{28)}$.

persons with a history of stroke at baseline or lack of retinal fundus information and/or vascular risk factors information. We also excluded cases that developed disabling dementia within 5 years from their baseline. The Ethics Committees of the Osaka Center for Cancer and Cardiovascular Disease Prevention and Osaka University approved this study.

\section{Retinal Vascular Changes}

All participants underwent retinal fundus photography of their right eye using a retinal camera at least once every 2 years. When a photograph of the right eye was not obtained due to anterior segment or media opacity (e.g., corneal opacity or cataracts), a photograph of the left eye was taken; photographs of the right eye were used in $96 \%$ of participants. Two well-trained physicians and/or medical technologists evaluated retinal findings and identified the presence of generalized arteriolar narrowing, focal arteriolar narrowing, arteriovenous nicking, increased arteriolar wall reflex, and any other retinopathy. As shown in Fig. 2, we graded retinal vascular change as "present" 
when the following criteria were found: (A) for generalized arteriolar narrowing, an arteriolar-to-venular ratio of 2:3 or lower; (B) for focal arteriolar narrowing, localized constrictions along the course of arterioles; (C) for arteriovenous nicking, narrowing or invisibility of a vein as an arteriole crossed over it; and (D) for increased arteriolar wall reflex, the presence of an increased light reflex from the central portion of the retinal arteriolar wall surface ${ }^{28)}$. The presence of other retinopathies was based on the observation of any of the following lesions: blot or flame-shaped hemorrhages, microaneurysms, exudates (soft or hard), optic disc swelling, new vessels at the disk or elsewhere, intra retinal microvascular abnormalities, vitreous hemorrhage, or laser photocoagulation scars ${ }^{28)}$. Additionally, the overall impact of retinal microvascular changes was estimated based on the total number of retinal abnormalities, including generalized arteriolar narrowing, focal arteriolar narrowing, arteriovenous nicking, increased arteriolar wall reflex, and the other retinopathies. We previously calculated intergrader prevalence- and bias-adjusted Kappa scores in a preliminary validation study of 116 eyes, and the principal major results were 0.52 for generalized arteriolar narrowing, 0.48 for focal arteriolar narrowing, 0.59 for arteriovenous nicking, and 0.98 for hemorrhages.

\section{Vascular Risk Factors}

We calculated body mass index as body weight $(\mathrm{kg})$ divided by body height squared $\left(\mathrm{m}^{2}\right)$, and overweight was defined as $25 \mathrm{~kg} / \mathrm{m}^{2}$ or higher. Blood pressures were measured by trained physicians using standard mercury sphygmomanometers and standardized epidemiological methods. We defined hypertension as systolic blood pressure of $140 \mathrm{mmHg}$ or higher and/ or diastolic blood pressure of $90 \mathrm{mmHg}$ or higher and/or antihypertensive medication use. We also identified hyperglycemia as fasting serum glucose of 110 $\mathrm{mg} / \mathrm{dl}$ or higher or casual serum glucose $140 \mathrm{mg} / \mathrm{dl}$ or greater and/or antidiabetic medication use and hypercholesterolemia as serum total cholesterol of $220 \mathrm{mg} /$ $\mathrm{dl}$ or higher and/or lipid-lowering medication use. A standard 12-lead resting electrocardiogram was obtained in supine position, and we noted atrial fibrillation (Minnesota Codes, 8-3-1 or 8-3-2) and ST-T changes (Minnesota Code, 4-1 to 4-3 and/or 5-1 to 5-3). We identified current smokers as those who reported smoking one or more cigarettes per day, past smokers as those who had quit smoking for 3 months or more, current drinkers as those who reported drinking 1 or more times per week, and ex-drinkers as those who had not drunk for 3 months or more.

\section{Ascertainment of Incidence of Stroke}

From 1981 to the present, we obtained information on incidence of stroke from death certificates, national insurance claims, annual cardiovascular risk surveys, and reports by local physicians, public health nurses, and health volunteers. To confirm the diagnoses, all living patients were telephoned, visited, or invited to participate in the risk surveys, or alternatively, a medical history was obtained from their families. In addition, medical records and findings of imaging studies such as CT and/or MRI from local clinics and hospitals were reviewed. In cases of death, histories were obtained from families and/or attending physicians and medical records were reviewed. The definition of incidence of stroke was a focal neurological disorder with rapid onset that persisted for at least $24 \mathrm{~h}$ or until death (International Classification of Diseases, 9 th Revision, code 430-438). Final diagnoses of stroke were made by a panel of three to four epidemiologists who were blinded to the data from the risk surveys.

\section{Statistical Analysis}

The analysis of covariance was used to test for differences in means and proportions of baseline characteristics between cases and controls. We calculated the conditional odds ratios (ORs) and 95\% confidence intervals ( $95 \%$ CIs) for incidence of disabling dementia associated with generalized arteriolar narrowing, focal arteriolar narrowing, arteriovenous nicking, increased arteriolar wall reflex, any other retinopathy, and the total number of retinal abnormalities (one, two, or more). Two models were constructed. Model 1 included adjustments for vascular risk factors: overweight status (yes, no), hypertension (yes, no), hyperglycemia (yes, no), hypercholesteremia (yes, no), electrocardiogram abnormality (yes, no), current smoking status (current, past, never), and drinking status (current, ex-, never). Model 2 incorporated full covariate adjustment, specifically adding an intermediate variable, namely the presence of incidence of stroke before the onset of disabling dementia (yes, no). SAS9.3 (SAS Institute Inc., Cary, NC) was used for analyses, and a two-tailed $p$ value of $<0.05$ denoted the presence of a statistically significant difference.

\section{Results}

The baseline characteristics of the 351 cases and 702 controls are shown in Table 1. Cases and controls showed no differences in overweight status, hypertension, hypercholesterolemia, or electrocardiogram abnormalities, but differed in terms of proportions of 
Table 1. Comparison of baseline characteristics between cases with disabling dementia and controls

\begin{tabular}{lcrc}
\hline & Case & Control & P-value \\
& $(\mathrm{N}=351)$ & $(\mathrm{N}=702)$ & 0.873 \\
\hline At baseline & & $67.8(0.3)$ & 1.000 \\
Age, years & $67.9(0.4)$ & $35.6(1.8)$ & 0.196 \\
Male, \% & $35.6(2.6)$ & $24.4(0.1)$ & 0.856 \\
Body mass index, kg/m ${ }^{2}$ & $24.1(0.2)$ & $15.2(1.4)$ & 0.676 \\
Overweight status, \% & $15.7(1.9)$ & $134.5(0.7)$ & 0.499 \\
Systolic blood pressure, mmHg & $135.0(0.9)$ & $78.5(0.4)$ & 0.894 \\
Diastolic blood pressure, mmHg & $78.9(0.5)$ & $59.1(1.9)$ & 0.429 \\
Hypertension, \% & $58.7(2.6)$ & $42.5(1.9)$ & 0.014 \\
Antihypertensive medication use, \% & $45.0(2.6)$ & $17.8(1.5)$ & 0.927 \\
Hyperglycemia, \% & $24.2(2.1)$ & $6.1(0.9)$ & 0.304 \\
Glucose-lowering medication use, \% & $6.0(1.3)$ & $204.3(1.3)$ & 0.344 \\
Total cholesterol, mg/dl & $202.0(1.8)$ & $38.0(1.8)$ & 0.617 \\
Hypercholesterolemia, \% & $35.0(2.6)$ & $10.7(1.2)$ & 0.179 \\
Lipid-lowering medication use, \% & $9.7(1.6)$ & $16.2(1.1)$ & 0.560 \\
Electrocardiogram abnormality, \% & $11.1(1.6)$ & $12.0(1.2)$ & 0.684 \\
Current smoker, \% & $17.7(2.0)$ & $28.1(1.7)$ & 0.550 \\
Past smoker, \% & $11.1(1.7)$ & $4.0(0.8)$ & 0.296 \\
Current drinker, \% & $30.0(2.4)$ & $12.1(1.4)$ & \\
Ex-drinker, \% & $5.4(1.1)$ & & \\
In followed-up period & & \\
Incidence of stroke, \% & $23.9(1.9)$ & & \\
\hline Men & & \\
\end{tabular}

Mean values (standard error); Body mass index, body weight $(\mathrm{kg})$ divided by squared body height $(\mathrm{m})$; Overweight status, Body mass index, $\geq 25 \mathrm{~kg} / \mathrm{m}^{2}$; Hypertension, systolic blood pressure $\geq 140 \mathrm{mmHg}$ and/or diastolic blood pressure $\geq 90 \mathrm{mmHg}$ and/or antihypertensive medication use; Hypercholesterolemia, serum total cholesterol $\geq 220 \mathrm{mg} / \mathrm{dl}$ and/or lipid-lowering medication use; Hyperglycemia, fasting serum glucose $\geq 110 \mathrm{mg} / \mathrm{dl}$ or casual serum glucose $\geq 140 \mathrm{mg} / \mathrm{dl}$ and/or glucose-lowering medication use; Electrocardiogram abnormality, atrial fibrillation (Minnesota Codes, 8-3-1 or 8-3-2) and/or ST-T changes (Minnesota Code, 4-1 to 4-3 and/or 5-1 to 5-3); Current smoking status (current, past, never) and drinking status (current, ex-, never).

hyperglycemia and incidence of stroke before the onset of disabling dementia.

The proportion of subjects with each retinal finding was as follows: generalized arteriolar narrowing, $18.0 \%$; arteriovenous nicking, $13.1 \%$; any other retinopathy, $10.3 \%$; increased arteriolar wall reflex, 9.6\%; and focal arteriolar narrowing, 7.9\%. As shown in Table 2, generalized arteriolar narrowing (case: $23.1 \%$ vs control: $15.7 \%, P=0.003)$ and two or more retinal abnormalities (case: $18.2 \%$ vs control: $13.4 \%$, $P=0.038$ ) were more frequent in cases compared with controls. While arteriovenous nicking tended to be more frequent in cases than in controls (case: 15.7\% vs control: $11.8 \%, P=0.081)$, the other conditions occurred at similar rates in cases and controls.

As shown in Table 3, we tested the associations between each retinal vascular change and disabling dementia using conditional logistic regression models. Generalized arteriolar narrowing was associated with an increased risk of disabling dementia: crude OR, 1.66 (95\% CI, 1.19-2.31), $P=0.003$. The positive association remained statistically significant after adjustment for vascular risk factors: OR, 1.58 (1.122.23), $P=0.010$ in Model 1 , and after further adjustment for incidence of stroke before the onset of disabling dementia: OR, $1.48(1.04-2.10), P=0.029$ in Model 2. Arteriovenous nicking was associated with borderline increased risk of disabling dementia: crude OR, 1.39 (0.96-2.01), $P=0.081$; OR, 1.39 (0.952.03), $P=0.087$ in Model 1; OR, 1.32 (0.90-1.95), $P=0.154$ in Model 2. Compared with no retinal abnormality, two or more retinal abnormalities were associated in a dose-response manner with an increased risk of disabling dementia even after adjustment for both vascular risk factors and incidence of stroke before the onset of disabling dementia. Other retinal vascular changes were not associated with increased risk.

\section{Discussion}

In this population-based nested case-control 
Table 2. Comparison of proportions of retinal microvascular abnormalities between cases with disabling dementia and controls at baseline

\begin{tabular}{lcrc}
\hline & $\begin{array}{c}\text { Case } \\
(\mathrm{N}=351)\end{array}$ & $\begin{array}{c}\text { Control } \\
(\mathrm{N}=702)\end{array}$ & P-value \\
\hline Generalized arteriolar narrowing, \% & $23.1(2.1)$ & $15.7(1.4)$ & 0.003 \\
Focal arteriolar narrowing, \% & $7.7(1.4)$ & $7.5(1.0)$ & 0.935 \\
Arteriovenous nicking, \% & $15.7(1.8)$ & $11.8(1.3)$ & 0.081 \\
Increased arteriolar wall reflex, \% & $10.5(1.6)$ & $9.3(1.1)$ & 0.508 \\
Any other retinopathy, \% & $11.4(1.6)$ & $9.8(1.2)$ & 0.432 \\
$\quad$ Hemorrhages, \% & $7.1(1.3)$ & $6.6(0.9)$ & 0.728 \\
$\quad$ Microaneurysms, \% & $2.6(0.7)$ & $1.4(0.5)$ & 0.191 \\
$\quad$ Exudates, \% & $2.8(0.9)$ & $2.6(0.6)$ & 0.787 \\
$\quad$ New vessels, \% & $1.1(0.5)$ & $0.9(0.4)$ & 0.654 \\
$\quad$ Laser photocoagulation scars, \% & $0.9(0.5)$ & $1.0(0.4)$ & 0.822 \\
Total number of retinal abnormalities & & & \\
$\quad 1$ finding, \% & $26.8(2.3)$ & $22.8(1.6)$ & 0.154 \\
$\geq 2$ findings, \% & $18.2(1.9)$ & $13.4(1.3)$ & 0.038 \\
\hline
\end{tabular}

In parentheses, standard error; Optic disc swelling, which is one of any other retinopathy, was not observed in this study population.

study, we found that a retinal vascular change, specifically generalized arteriolar narrowing i.e., a low ratio of arteriolar-to-venular diameter (2:3 or lower), was associated with an increased risk of disabling dementia, and the risk was unchanged after adjustment for vascular risk factors. While arteriovenous nicking showed a nonsignificant association with the risk, the other retinal abnormalities demonstrated no association. The total number of retinal abnormalities was also associated with the risk in a dose-response manner.

Previous longitudinal population-based studies have shown similar results linking retinal vascular changes to various indicators of incidence of dementia ${ }^{11-13,17)}$. According to an 11.6-year follow-up of men and women aged 55 years and older in the Rotterdam Study, generalized arteriolar narrowing and venular dilation tended to be associated with incidence of dementia ${ }^{9)}$, although the study did not report arteriolar-to-venular ratios, which we used as a criterion for the diagnosis of generalized arteriolar narrowing. Their findings seem to be consistent with those of the present study because small arteriolar diameter and the venular dilation led to small arteriolar-to-venular ratio. In the Rotterdam Study, Ikram et al. found that venular dilation and arteriolar-to-venular ratio were associated with the progression of brain abnormalities detected by imaging, namely periventricular white matter lesions and lacunar infarcts over 3 years, whereas only venular dilation was strongly associated with the progression of subcortical white matter lesions ${ }^{12)}$. Schrijvers et al. also reported a positive association between retinopathy (hemorrhages, microan- eurysms, cotton wool spots, and laser photocoagulation scars) and the prevalence of dementia in a crosssectional analysis, but no association with the risk of dementia in a prospective analysis ${ }^{13)}$. In a 5 -year follow-up of men and women aged 65 years and older of the Cardiovascular Health Study, they reported doseresponse relationships between the total number of retinal vascular changes and incidence of disability, defined by executive dysfunction, slow gait, and depressive symptoms ${ }^{17)}$. These results support the present finding that retinal vascular changes, represented by a small arteriolar-to-venular ratio, may help predict the risk of incidence of disabling dementia.

A 14-year follow-up of men and women aged 45-64 years of the Atherosclerosis Risk in Communities (ARIC) study did not investigate the association between retinal vascular changes and the risk of dementia, but it did examine the association between this risk and cognitive decline ${ }^{16)}$. The ARIC study found that cognitive decline was associated with focal arteriolar narrowing and retinopathy but not generalized arteriolar narrowing. They did not state the reason for the lack of correlation with generalized arteriolar narrowing. However, cognitive decline is not always a preclinical sign of dementia, and these two outcomes likely have different predictors ${ }^{10)}$.

As previous studies have reported the associations of hypertension with generalized arteriolar narrowing $^{29)}$ and incidence of dementia ${ }^{30)}$, hypertension could be one of the most important risk factors in the present study. However, we found no association between hypertension and incidence of disabling dementia among the subjects (average age $=68$ years). 
Table 3. Conditional odds ratios for incidence of disabling dementia according to each retinal microvascular abnormality

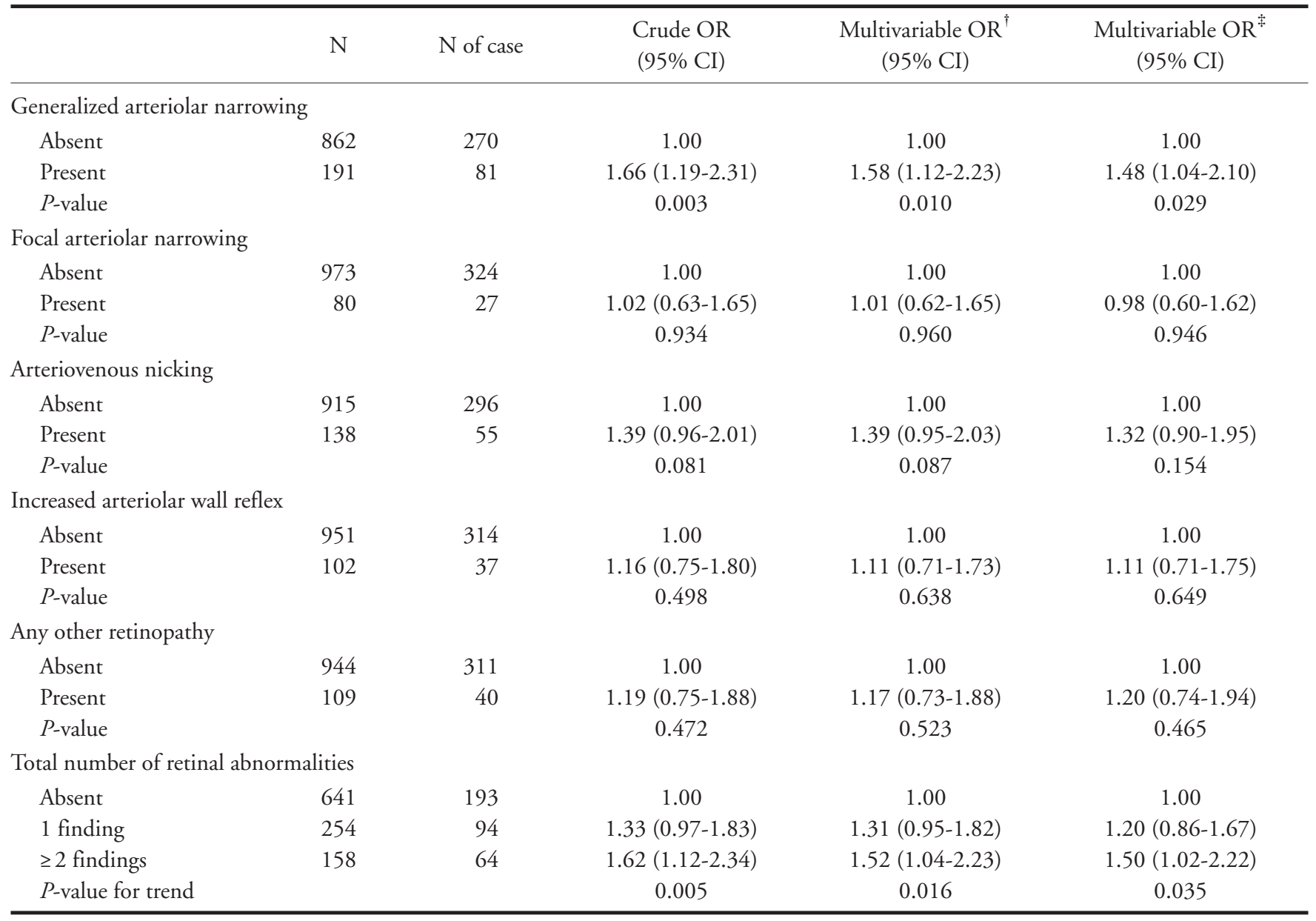

OR, conditional odds ratio; 95\% CI, 95\% confidence interval; Case-control matching variables: sex, age ( \pm 3 years), and baseline-year.

Adjustment for vascular risk factors included overweight status, hypertension, hyperglycemia, hypercholesterolemia, electrocardiogram abnormality, current smoking status (current, past, never) and drinking status (current, ex-, never).

F Further adjustment for incidence of stroke before the onset of disabling dementia.

The contribution of hypertension to the development of dementia may be weakened among the elderly. Ninomiya et al. reported the association of hypertension with incidence of all-cause dementia among middle-aged adults (average age $=57$ years), but not among older adults (average age $=72$ years) ${ }^{30}$. In addition, generalized arteriolar narrowing, associated with the risk of incidence of disabling dementia in our study, was likely to be caused by long-term hyperten$\operatorname{sion}^{31)}$. On the other hand, focal arteriolar narrowing, which was not associated with the risk of incidence of disabling dementia in our study, was reported to be caused by short-term hypertension ${ }^{31)}$. Thus, long-term hypertension from young to middle age may cause generalized arteriolar narrowing, and it may lead to incidence of disabling dementia in the elderly.

The most important strength of our study was the longitudinal population-based design. No other studies in Asia have shown the associations between comprehensive retinal vascular changes and the risk of disabling dementia. Some further methodologic issues should be discussed. We used a subjective grading system to evaluate retinal vascular changes. Such methods generally show lower reproducibility than computer-assisted systems ${ }^{32}$. However, the significant associations in the present study indicate that standardized qualitative grading is also useful in identifying persons at higher risks of disabling dementia.

\section{Conclusion}

We found that retinal vascular changes were positively associated with the risk of disabling dementia, even after adjustment for vascular risk factors and incidence of stroke before the onset of disabling dementia. Generalized arteriolar narrowing and total 
number of retinal abnormalities may be useful markers for identifying persons at higher risks of disabling dementia.

\section{CIRCS Investigators}

The CIRCS Investigators are: Isao Muraki, Mina Hayama-Terada, Shinichi Sato, Yuji Shimizu, Takeo Okada, and Masahiko Kiyama, Osaka Center for Cancer and Cardiovascular Disease Prevention; Akihiko Kitamura, Hironori Imano, Renzhe Cui and Hiroyasu Iso, Osaka University; Kazumasa Yamagishi and Tomoko Sankai, University of Tsukuba; Isao Koyama and Masakazu Nakamura, National Cerebral and Cardiovascular Center; Masanori Nagao and Mitsumasa Umesawa, Dokkyo Medical University School of Medicine; Tetsuya Ohira, Fukushima Medical University; Isao Saito, Ehime University; and Ai Ikeda, Koutatsu Maruyama and Takeshi Tanigawa, Juntendo University.

\section{Acknowledgements}

The authors thank Professor Emeritus Yoshio Komachi (University of Tsukuba), Professor Emeritus Hideki Ozawa (Oita Medical University), Former professor Minoru Iida (Kansai University of Welfare Sciences), Professor Emeritus Takashi Shimamoto (University of Tsukuba), Dr Yoshinori Ishikawa (Consultant of Osaka Center for Cancer and Cardiovascular Disease Prevention), Professor Yoshihiko Naito (Mukogawa Women's University), Professor Tomonori Okamura (Keio University) for their support in conducting long-term cohort studies, and Drs Ai Ikeda, Hiroyuki Noda, Choy-Lye Chei for their valuable comments and data correction. The authors also thank the clinical laboratory technologists, public health nurses, engineers of the computer processing unit, nurses, and nutritionists in the Osaka Center for Cancer and Cardiovascular Disease Prevention and health professionals in the survey communities for their valuable assistance for their expert help.

\section{Sources of Funding}

This study was supported in part by Grants-inAid for Scientific (Research A, 26253043; Research C, 26460791), funded by Japan Society for the Promotion of Science, and by Health and Labour Science Research Grants for Dementia (H21-NinchishoWakate-007; H24-Ninchisho-Wakate-003), funded by Ministry of Health, Labour and Welfare, Japan.

\section{Conflict of Interest}

None.

\section{References}

1) Etters L, Goodall D, Harrison BE: Caregiver burden among dementia patient caregivers: a review of the literature. J Am Acad Nurse Pract, 2008; 20: 423-428

2) Muraki I, Yamagishi K, Ito $Y$, Fujieda $T$, Ishikawa $Y$, Miyagawa Y, Okada K, Sato S, Kitamura A, Shimamoto $\mathrm{T}$, Tanigawa $\mathrm{T}$, Iso $\mathrm{H}$ : Caregiver burden for impaired elderly Japanese with prevalent stroke and dementia under long-term care insurance system. Cerebrovasc Dis, 2008; 25: $234-240$

3) Gorelick PB, Scuteri A, Black SE, Decarli C, Greenberg SM, Iadecola C, Launer LJ, Laurent S, Lopez OL, Nyenhuis D, Petersen RC, Schneider JA, Tzourio C, Arnett DK, Bennett DA, Chui HC, Higashida RT, Lindquist R, Nilsson PM, Roman GC, Sellke FW, Seshadri S: Vascular contributions to cognitive impairment and dementia: a statement for healthcare professionals from the American Heart Association/American Stroke Association. Stroke, 2011; 42: 2672-2713

4) de la Torre JC: Impaired brain microcirculation may trigger Alzheimer's disease. Neurosci Biobehav Rev, 1994; 18: 397-401

5) Yukutake T, Yamada $M$, Fukutani $N$, Nishiguchi $S$, Kayama $\mathrm{H}$, Tanigawa $\mathrm{T}$, Adachi $\mathrm{D}$, Hotta $\mathrm{T}$, Morino $\mathrm{S}$, Tashiro Y, Arai H, Aoyama T. Arterial stiffness determined according to the cardio-ankle vascular index (CAVI) is associated with mild cognitive decline in communitydwelling elderly subjects. J Atheroscler Thromb, 2014; 21: 49-55

6) Yukutake T, Yamada M, Fukutani N, Nishiguchi $S$, Kayama $\mathrm{H}$, Tanigawa $\mathrm{T}$, Adachi $\mathrm{D}$, Hotta $\mathrm{T}$, Morino $\mathrm{S}$, Tashiro Y, Aoyama T, Arai H. Arterial stiffness predicts cognitive decline in Japanese community-dwelling elderly subjects: a one-year follow-up study. J Atheroscler Thromb, 2015; 22: 637-644

7) Wong TY, McIntosh R: Systemic associations of retinal microvascular signs: a review of recent population-based studies. Ophthalmic Physiol Opt, 2005; 25: 195-204

8) Patton N, Aslam T, Macgillivray T, Pattie A, Deary IJ, Dhillon B: Retinal vascular image analysis as a potential screening tool for cerebrovascular disease: a rationale based on homology between cerebral and retinal microvasculatures. J Anat, 2005; 206: 319-348

9) Perez MA, Bruce BB, Newman NJ, Biousse V: The use of retinal photography in nonophthalmic settings and its potential for neurology. Neurologist, 2012; 18: 350-355

10) Heringa SM, Bouvy WH, van den Berg E, Moll AC, Kappelle LJ, Biessels GJ: Associations between retinal microvascular changes and dementia, cognitive functioning, and brain imaging abnormalities: a systematic review. J Cereb Blood Flow Metab, 2013; 33: 983-995

11) de Jong FJ, Schrijvers EM, Ikram MK, Koudstaal PJ, de Jong PT, Hofman A, Vingerling JR, Breteler MM: Retinal vascular diameter and risk of dementia: the Rotterdam study. Neurology, 2011; 76: 816-821 
12) Ikram MK, De Jong FJ, Van Dijk EJ, Prins ND, Hofman A, Breteler MM, De Jong PT: Retinal vessel diameters and cerebral small vessel disease: the Rotterdam Scan Study. Brain, 2006; 129: 182-188

13) Schrijvers EM, Buitendijk GH, Ikram MK, Koudstaal PJ, Hofman A, Vingerling JR, Breteler MM: Retinopathy and risk of dementia: the Rotterdam Study. Neurology, 2012; 79: 365-370

14) Kawasaki R, Cheung N, Mosley T, Islam AF, Sharrett AR, Klein R, Coker LH, Knopman DS, Shibata DK, Catellier D, Wong TY: Retinal microvascular signs and 10-year risk of cerebral atrophy: the Atherosclerosis Risk in Communities (ARIC) study. Stroke, 2010; 41: 1826-1828

15) Cheung N, Mosley T, Islam A, Kawasaki R, Sharrett AR, Klein R, Coker LH, Knopman DS, Shibata DK, Catellier D, Wong TY: Retinal microvascular abnormalities and subclinical magnetic resonance imaging brain infarct: a prospective study. Brain, 2010; 133: 1987-1993

16) Lesage SR, Mosley TH, Wong TY, Szklo M, Knopman D, Catellier DJ, Cole SR, Klein R, Coresh J, Coker LH, Sharrett AR: Retinal microvascular abnormalities and cognitive decline: the ARIC 14-year follow-up study. Neurology, 2009; 73: 862-868

17) Kim DH, Chaves PH, Newman AB, Klein R, Sarnak MJ, Newton E, Strotmeyer ES, Burke GL, Lipsitz LA: Retinal microvascular signs and disability in the Cardiovascular Health Study. Arch Ophthalmol, 2012; 130: 350-356

18) Wong TY: Is retinal photography useful in the measurement of stroke risk. Lancet Neurol, 2004; 3: 179-183

19) Mimoun L, Massin P, Steg G: Retinal microvascularisation abnormalities and cardiovascular risk. Arch Cardiovasc Dis, 2009; 102: 449-456

20) Henderson AD, Bruce BB, Newman NJ, Biousse V: Hypertension-related eye abnormalities and the risk of stroke. Rev Neurol Dis, 2011; 8: 1-9

21) Ikeda A, Yamagishi K, Tanigawa T, Cui R, Yao M, Noda H, Umesawa M, Chei C, Yokota K, Shiina Y, Harada M, Murata K, Asada T, Shimamoto T, Iso H: Cigarette smoking and risk of disabling dementia in a Japanese rural community: a nested case-control study. Cerebrovasc Dis, 2008; 25: 324-331

22) Imano $H$, Kitamura $A$, Sato $S$, Kiyama $M$, Ohira $T$, Yamagishi $\mathrm{K}$, Noda $\mathrm{H}$, Tanigawa $\mathrm{T}$, Iso $\mathrm{H}$, Shimamoto $\mathrm{T}$ : Trends for blood pressure and its contribution to stroke incidence in the middle-aged Japanese population: the
Circulatory Risk in Communities Study (CIRCS). Stroke, 2009; 40: 1571-1577

23) Imano $H$, Iso $H$, Kiyama $M$, Yamagishi $K$, Ohira $T$, Sato S, Noda H, Maeda K, Okada T, Tanigawa T, Kitamura A; CIRCS Investigators: Non-fasting blood glucose and risk of incident coronary heart disease in middle-aged general population: the Circulatory Risk in Communities Study (CIRCS). Prev Med, 2012; 55: 603-607

24) Chei CL, Yamagishi K, Ikeda A, Noda H, Maruyama M, Cui R, Imano H, Kiyama M, Kitamura A, Asada T, Iso H; CIRCS Investigators: C-reactive protein levels and risk of disabling dementia with and without stroke in Japanese: the Circulatory Risk in Communities Study (CIRCS). Atherosclerosis, 2014; 236: 438-443

25) Yamagishi K, Ikeda A, Moriyama Y, Chei CL, Noda H, Umesawa M, Cui R, Nagao M, Kitamura A, Yamamoto Y, Asada T, Iso H; CIRCS Investigators: Serum coenzyme Q10 and risk of disabling dementia: the Circulatory Risk in Communities Study (CIRCS). Atherosclerosis, 2014; 237: 400-403

26) Yamagishi K, Asada T. A prospective study of potential risk and protective factors for disabling dementia. Health and Labour Science Research Grants for Dementia (H21Ninchisho-Wakate-007, in Japanese), 2010: 15-23

27) Levy R: Aging-associated cognitive decline. Working Party of the International Psychogeriatric Association in collaboration with the World Health Organization. Int Psychogeriatr, 1994; 6: 63-68

28) Iida M, Kitamura A: Usefulness of non-mydriasis fundus photography in health checkup. 2nd Ed. Tokyo: Vector Core, 2009

29) Wong TY, Mitchell P: Hypertensive retinopathy. N Engl J Med, 2004; 351: 2310-2317

30) Ninomiya T, Ohara T, Hirakawa Y, Yoshida D, Doi Y, Hata J, Kanda S, Iwaki T, Kiyohara Y: Midlife and latelife blood pressure and dementia in Japanese elderly. Hypertension, 2011; 58: 22-28

31) Wong TY, McIntosh R: Systemic associations of retinal microvascular signs: a review of recent population-based studies. Ophthal Physiol Opt, 2005; 25: 195-204

32) Couper DJ, Klein R, Hubbard LD, Wong TY, Sorlie PD, Cooper LS, Brothers RJ, Nieto FJ: Reliability of retinal photography in the assessment of retinal microvascular characteristics: the Atherosclerosis Risk in Communities Study. Am J Ophthalmol, 2002; 133: 78-88 